Note

\title{
Correlation between Plasma $a$-Cysteine Proteinase Inhibitor Level and Pyruvate Kinase Activity in Vitamin E-Deficient Rats
}

\author{
Naoko HaRada, ${ }^{1}$ Shin-ichi Saito, ${ }^{1}$ and Kayoko MinaKata ${ }^{2}$ \\ ${ }^{1}$ University of Shizuoka, Hamamatsu College, 3-2-3, Nunohashi, \\ Hamamatsu 432, Japan \\ ${ }^{2}$ Department of Legal Medicine, Hamamatsu University, School of Medicine, \\ 3600 Handa, Hamamatsu 431-31, Japan
}

(Received February 24, 1992)

Summary Weaning rats were divided into two groups, one group being fed a vitamin E-deficient diet, and the other an $\alpha$-tocopherolcontaining $(50 \mathrm{mg} / \mathrm{kg})$ control diet. Rats were killed at $1,2,3$ and 4 months of feeding.

The following results were obtained.

1. Both plasma and liver $\alpha$-tocopherol levels decreased greatly by feeding vitamin E-deficient diet for 1 month, and thereafter decreased gradually by continued feeding.

2. Somehow different results were obtained concerning liver peroxidation value by the method of analysis. In the case of chemiluminescence, the value increased by vitamin $\mathrm{E}$ deficiency during the first 2 months, but thereafter, the value was almost unchanged. On the contrary, in the case of TBA-RS, the value increased gradually throughout the entire 4 months of feeding period.

3. Both plasma $\alpha$-CPI level and pyruvate kinase activity increased by vitamin $\mathrm{E}$ deficiency, showing similar pattern of change with feeding period. Especially, marked increase of these values was observed in vitamin E-deficient rats fed for longer than 2 months, and differences from control groups were highly significant (in both cases, at 2 months, $p<0.005$, and at 3 and 4 months, $p<0.001$ ). And, in vitamin E-deficient group, including all the rats fed on test diet for 1 to 4 months, correlation between both values was very high, and was highly significant $(r=0.9060$, $p<0.001$ ).

Key Words vitamin E, proteinase inhibitor, pyruvate kinase, lipid peroxide

Increase of plasma pyruvate kinase activity in vitamin E-deficient rats was made clear by Chow (1), and this increase was shown to be correlated well with the number of necrotized muscle fibers by Machlin et al. (2). 
In a dystrophic muscle caused by vitamin $\mathrm{E}$ deficiency, a significant increase was displayed by lysosomal proteinases and other hydrolases (3-6). Recently, inhibitory effects of low molecular weight proteinase inhibitors on the protein degeneration in muscular dystrophy were reported $(7,8)$. We have previously investigated the influence of vitamin $\mathrm{E}$ deficiency on two endogeneous proteinase inhibitors in plasma or serum, $\alpha$-cysteine proteinase inhibitor $\left(\alpha\right.$-CPI) and $\alpha_{1}$ proteinase inhibitor $\left(\alpha_{1}-\mathrm{PI}\right)$, and found that the former was increased greatly in vitamin E-deficient muscular dystrophic rats while the latter was almost unchanged (9).

Plasma $\alpha$-CPI is very stable and is unchanged under repeated freezing and thawing, and only a minute amount of sample is needed for its measurement (10). Therefore, the use of plasma $\alpha$-CPI level as a routine index of occurrence of muscular dystrophy in vitamin E-deficient animal would be very convenient. For this purpose, correlation between plasma $\alpha$-CPI level and pyruvate kinase activity in vitamin E-deficient animals has been investigated.

Materials and methods. Materials: All chemicals and reagents used were the same as described in our previous report (11).

Experimental design: Male rats of Wistar strain (Nihon SLC, Hamamatsu) were purchased after weaning. They were housed in individual cages in a temperature-controlled room $\left(22^{\circ} \mathrm{C}\right)$ with equal light and dark cycles daily.

Composition of vitamin E-deficient diet was the same as described in our previous report (11). In vitamin E-containing control diet, $50 \mathrm{mg}$ of all-rac- $\alpha$ tocopherol per $\mathrm{kg}$ of diet was added.

Eighty-two rats were purchased, and 11 rats were sacrificed immediately as base line for initial value, and remaining rats were divided into two groups: one group was given a vitamin E-deficient diet (41 rats), and the other a vitamin E-supplemented control diet (30 rats). At 1, 2, 3 and 4 months after initiation of feeding with test diet, a group of 7 or more rats from each group were sacrificed for the analysis.

Rats were killed by heart puncture under light Nembutal anesthesia and blood was collected by heparinized syringe, and plasma was separated by centrifugation. Then, liver was separated. Plasma pyruvate kinase activity was measured during the same day rats were sacrificed, and later plasma $\alpha$-CPI level was measured on frozen samples. Liver peroxidation values and plasma and liver $\alpha$-tocopherol levels were measured on frozen samples stored in a freezer at $-80^{\circ} \mathrm{C}$.

The determination of $\alpha$-tocopherol content in plasma and liver: These values were determined by the same way as described in our previous report (11).

Measurement of liver peroxidation value: Liver peroxidation value was measured by the two methods as described in our previous report (11).

Statistical analysis: The significance of the difference between two groups of values was determined by Student's $t$-test (12). And, as to correlation coefficient, level of statistical significance was also assessed by $t$-test (13).

Results and discussion. Plasma $\alpha$-CPI level and pyruvate kinase activity: 


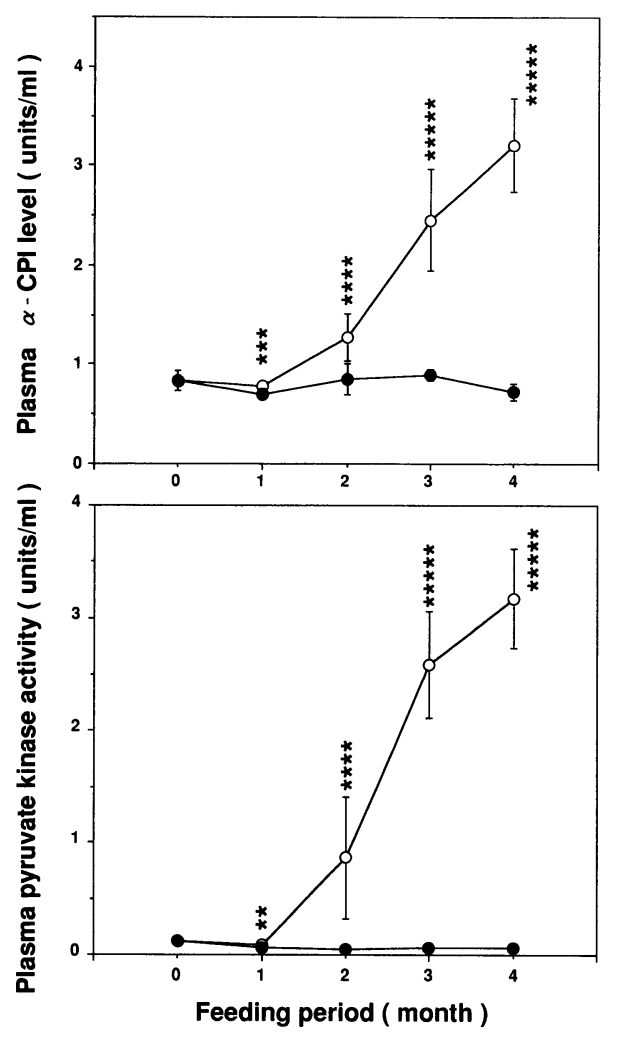

Fig. 1. Changes of plasma $\alpha$-CPI level and pyruvate kinase activity with feeding period in vitamin E-deficient and control diet-fed rats. Open and closed circles represent mean values of vitamin E-deficient and control groups, respectively, and bars represent standard deviation. Numbers of rats in each point are as follows: initial, 11 rats, for vitamin E-deficient group, at 1 month, 7 rats, at 2 months, 8 rats, at 3 months, 9 rats and at 4 months, 17 rats; and for control group, at 1 month, 7 rats, at 2 months, 7 rats, at 3 months, 9 rats and at 4 months, 7 rats. Asterisks $(*)$ represent significant differences between vitamin E-deficient and control groups of same feeding period; ${ }^{* *} p<0.025$, ${ }^{* * *} p<0.010$, $* * * * p<0.005, * * * * * p<0.001$.

Figure 1 shows the changes of plasma $\alpha$-CPI level and pyruvate kinase activity with feeding period in vitamin E-deficient and control groups. It can be seen from this figure that both values changed similarly with progression of vitamin E deficiency, although degree of increase was greater in the case of pyruvate kinase activity.

During 1 to 4 months of feeding with vitamin E-deficient diet, both values increased greatly, especially marked changes were observed during 2 to 3 months period. And, after 2 months, in both cases, the differences from control groups were always highly significant (in both cases, at 2 months, $p<0.005$, at 3 and 4 


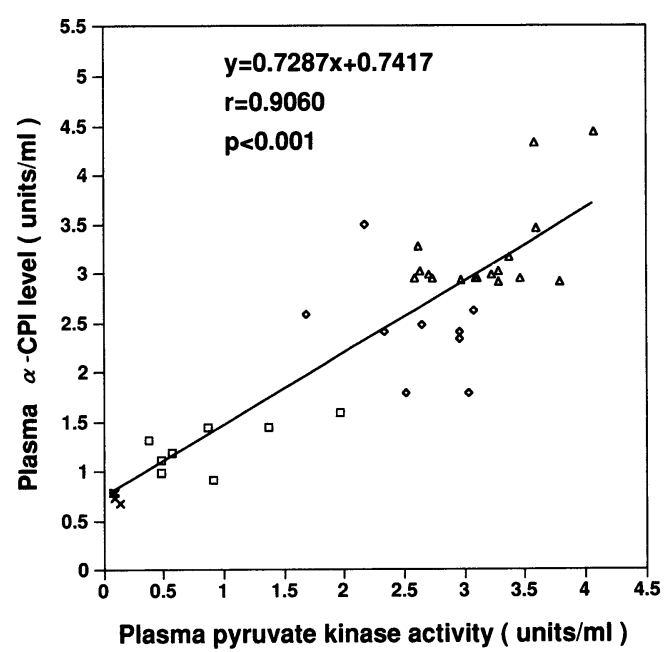

Fig. 2. Correlation between plasma $\alpha$-CPI level and pyruvate kinase activity in vitamin E-deficient diet-fed rats. Symbols are as follows: cross for rats fed on test diet for 1 month, square for 2 months, rhomb for 3 months and triangle for 4 months.

months, $p<0.001)$.

Correlation between $\alpha-C P I$ level and pyruvate kinase activity in vitamin $E$ deficient rats: Figure 2 shows the correlation between plasma $\alpha$-CPI level and pyruvate kinase activity in vitamin E-deficient diet-fed rats for 1 to 4 months. A very close correlation is observed between both values $(p<0.001)$.

Plasma and liver $\alpha$-tocopherol levels: Figure 3 shows the changes of plasma and liver $\alpha$-tocopherol levels in logarithmic scale with feeding period in vitamin E-deficient and control groups. Both values in vitamin E-deficient group decreased greatly at 1 month feeding period, and decreased thereafter gradually. The same tendency was also reported by other investigators (14-16).

Liver peroxidation value: Figure 4 shows the peroxidation value of the liver measured by the two different methods. In the case of chemiluminescence, in vitamin E-deficient group, the value increased greatly during 1 to 2 months of feeding period, but thereafter was almost unchanged. On the contrary, in the case of TBA-RS, the value of vitamin E-deficient group increased gradually throughout the 4 months of feeding period. Chemiluminescence is produced directly from active oxygen such as singlet oxygen, but TBA-RS is the secondary product from lipid peroxide. Wright et al. (17) showed in in vitro experiment for measurement of lipid peroxide formation using liver microsome that chemiluminescence reached maximum after incubation for some minutes, but MDA increased gradually after chemiluminescence began to decrease, indicating MDA as the accumulated secondary product.

Anyway, it is clear from Figs. 3 and 4 that in rats fed the vitamin E-deficient 


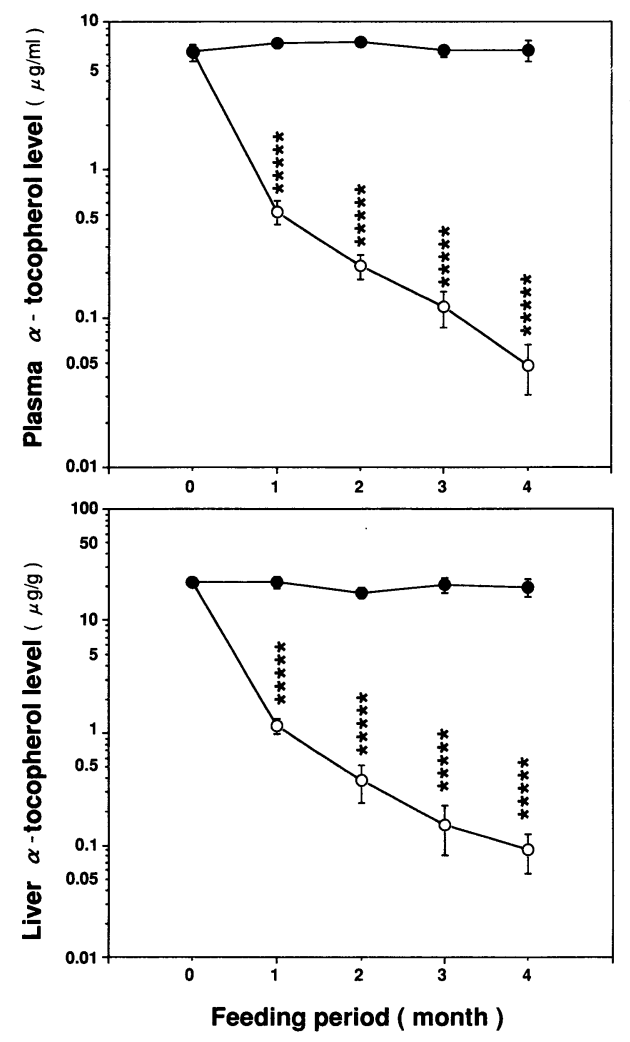

Fig. 3. Changes of plasma and liver $\alpha$-tocopherol levels with feeding period in vitamin E-deficient and control diet-fed rats. Symbols and numbers of rats in each point are the same as those in Fig. 1. Asterisks $(*)$ represent significant differences between vitamin E-deficient and control groups of same feeding period; $* * * * * p<0.001$.

diet for longer than 2 months, plasma and liver $\alpha$-tocopherol levels were very low, and peroxidation product accumulated, indicating progression of vitamin $\mathrm{E}$ deficiency to severe stage.

From the fact that in vitamin E-deficient rats high correlation between plasma $\alpha$-CPI level and pyruvate kinase activity was observed (Fig. 2), it would be reasonable to suppose that plasma $\alpha$-CPI level would be adequate as a routine index of occurrence of vitamin E deficiency symptoms such as muscular dystrophy, together with pyruvate kinase activity.

In the case of plasma pyruvate kinase activity, Machlin et al. (2), in recovery experiment from vitamin E deficiency using rats, postulated that increase of plasma pyruvate kinase activity reflects the number of necrotized muscle fibers, but it would not have direct relationships with testis or liver degeneration. In the case of $\alpha$-CPI, in testis of vitamin E-deficient rats, an increase of cysteine proteinase 

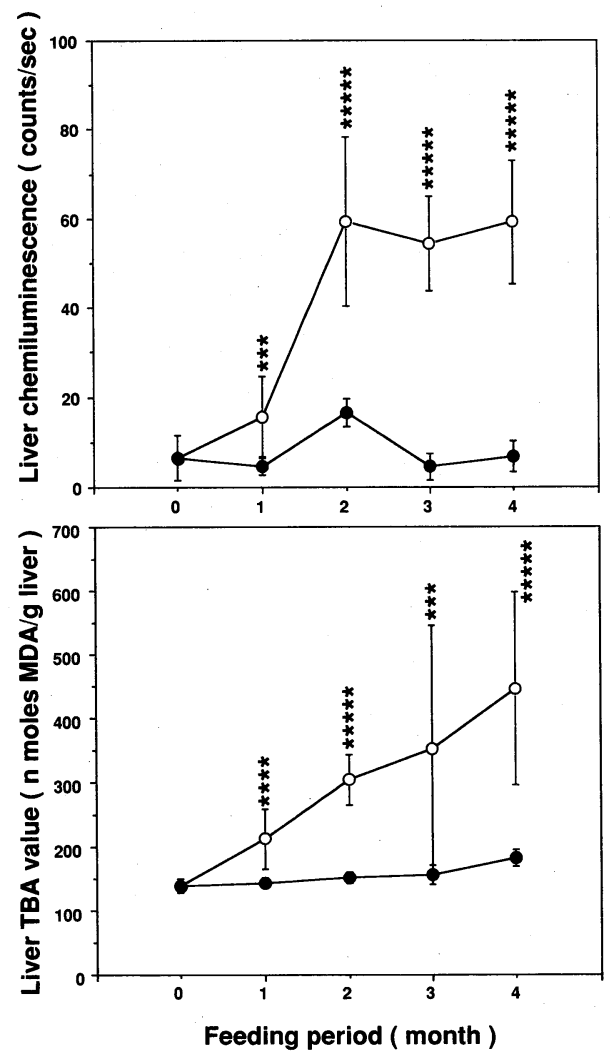

Fig. 4. Changes of peroxidation value of the liver measured by chemiluminescence and TBA method with feeding period in vitamin E-deficient and control diet-fed rats. Symbols and numbers of rats in each point are the same as those in Fig. 1. Asterisks $(*)$ represent significant differences between vitamin E-deficient and control groups of same feeding period; ${ }^{* * *} p<0.010,{ }^{* * * *} p<0.005$, $* * * * * p<$ 0.001 .

inhibitor level was concomitant with degeneration of tissue (18). Therefore, increase of plasma $\alpha$-CPI level would have some relationships not only with dystrophic muscle, but also with other tissues and organs including testis which were subjected to degeneration by vitamin E deficiency. The relationship in $\alpha$-CPI between plasma and testis remains to be investigated.

Recently, plasma cysteine proteinase inhibitors are shown to be identical to kininogens $(19,20)$. Three kinds of kininogens are known to be present in plasma, namely, HMW-, LMW- and T-kininogens. In rat, increase of T-kininogen was reported in various cases such as inflammation (21-23). Whether plasma $\alpha$-CPI increased by vitamin E deficiency is identical to HMW-, LMW- or to T-kininogen also remains to be solved. 


\section{REFERENCES}

1) Chow, C. K. (1975): Increased activity of pyruvate kinase in plasma of vitamin E-deficient rats. J. Nutr., 105, 1221-1224.

2) Machlin, L. J., Gabriel, E., Spiegel, H. E., Horn, L. R., Brin, M., and Nelson, J. (1978): Plasma activity of pyruvate kinase and glutamic oxalacetic transaminase as indices of myopathy in the vitamin E deficient rats. J. Nutr., 108, 1963-1968.

3) Weinstock, I. M., Goldrich, A. D., and Milhort, A. T. (1956): Enzyme studies in muscular dystrophy II. Muscle dipeptidase activity and vitamin E deficiency. Proc. Soc. Exp. Biol. Med., 91, 302-305.

4) Zalkin, H., Tappel, A. L., Caldwell, K. A., Shibko, S., Desai, I. D., and Holliday, T. A. (1962): Increased lysosomal enzymes in muscular dystrophy of vitamin E-deficient rabbits. J. Biol. Chem., 237, 2678-2682.

5) Bunyan, J., Green, J., and Diplock, A. T. (1967): Lysosomal enzymes and vitamin E deficiency. I. Muscular dystrophy, encephalomalacia and exudative diathesis in chick. Br. J. Nutr., 21, 127-136.

6) Dayton, W. R., Schollmeyer, J. V., Chan, A. C., and Allen, C. E. (1979): Elevated levels of a calcium-activated muscle proteinase in rapidly atrophying muscle from vitamin E-deficient rabbits. Biochim. Biophys. Acta, 584, 216-230.

7) Libby, P., and Goldberg, A. L. (1978): Leupeptin, a proteinase inhibitor, decreases protein degeneration in normal and diseased muscles. Science, 199, 534-536.

8) Stracher, A., Mcgowan, E. B., and Shafiq, S. A. (1978): Muscular dystrophy. Inhibition of degeneration in vivo with proteinase inhibitors. Science, 200, 50-51.

9) Minakata, K., Asano, M., Takahashi, Y., and Harada, N. (1984): Increase of $\alpha$-cysteine proteinase inhibitor level in serum of vitamin $\mathrm{E}$ deficient muscular dystrophic rats. Nutr. Rep. Int., 29, 445-459.

10) Minakata, K., Asano, M., Sato, T., and Harada, N. (1982): Assay of $\alpha$-cysteine proteinase inhibitor in serum or plasma. Hoppe-Seyler's Z. Physiol. Chem., 363, 493498.

11) Harada, N., Saito, S., and Minakata, K. (1991): Effects of vitamin E on toxicity by minute amounts of paraquat fed continuously to rats. J. Nutr. Sci. Vitaminol., 37, 113.

12) Snedecor, G. W., and Cochran, W. G. (1967): Statistical Methods, 6th ed., Iowa State Univ. Press, Ames, Chapter 4.

13) Terada, K. (1973): Suisokutoukeihou (in Japanese), Asakurashoten, Tokyo, pp. 133 -141 .

14) Machlin, L. J., Keating, J., Nelson, J., Brin, M., Fillipski, R., and Miller, O. N. (1979): Availability of adipose tissue tocopherol in the guinea pig. J. Nutr., 109, 105-109.

15) Mino, M., Kitagawa, M., and Nakagawa, S. (1981): Changes of alpha-tocopherol levels in red blood cells and plasma with respect to hemolysis induced by dialuric acid in vitamin E-deficient rats. J. Nutr. Sci. Vitaminol., 27, 199-207.

16) Bourre, J., and Clement, M. (1991): Kinetics of rat peripheral nerve, forebrain, and cerebellum $\alpha$-tocopherol depletion: Comparison with different organs. J. Nutr., 121, 1204-1207.

17) Wright, J. R., Rumbaugh, R. C., Colby, H. D., and Miles, P. R. (1979): The 
relationship between chemiluminescence and lipid peroxidation in rat hepatic microsomes. Arch. Biochem. Biophys., 192, 344-351.

18) Minakata, K., Asano, M., Takahashi, Y., and Harada, N. (1987): Enhancement of testicular cysteine proteinase inhibitor level in vitamin E-deficient rats. J. Nutr., 117, 1416-1421.

19) Ohkubo, I., Kurachi, K., Takasawa, T., Shiokawa, H., and Sasaki, M. (1984): Isolation of a human cDNA for $\alpha_{2}$-thiol proteinase inhibitor and its identity with low molecular weight kininogen. Biochemistry, 23, 5691-5697.

20) Sueyoshi, T., Enjyoji, K., Shimada, T., Kato, H., Iwanaga, S., Bando, Y., Kominami, E., and Katsunuma, N. (1985): A new function of kininogens as thiol-proteinase inhibitors: Inhibition of papain and cathepsin $\mathrm{B}, \mathrm{H}$ and $\mathrm{L}$ by bovine, rat and human plasma kininogens. FEBS Lett., 182, 193-195.

21) Barlas, A., Okamoto, H., and Greenbaum., L. M. (1985): T-kininogen-The major plasma kininogen in rat adjuvant arthritis. Biochem. Biophys. Res. Commun., 129, 280-286.

22) Sakamoto, W., Yoshikawa, K., Handa, H., Uehara, S., and Hirayama, A. (1986): T-kininogen in rats with carrageenin-induced inflammation. Biochem. Pharmacol., 35, 4283-4290.

23) Sakamoto, W., and Mino, M. (1991): Vitamin E and macrophage. Vitamins (in Japanese), 65, 289-300. 